\title{
Évolution du système financier rural à Java -Indonésie-
} Sandrine Dury, Cécile Lapenu

\section{Citer ce document / Cite this document :}

Dury Sandrine, Lapenu Cécile. Évolution du système financier rural à Java -Indonésie-. In: Économie rurale. N²27, 1995. pp. 34-43;

doi : https://doi.org/10.3406/ecoru.1995.4732

https://www.persee.fr/doc/ecoru_0013-0559_1995_num_227_1_4732

Fichier pdf généré le 08/05/2018 


\title{
Résumé
}

A travers des études de cas les auteurs décrivent la diversité et le rôle des secteurs formel et informel du système financier indonésien en milieu rural. L'analyse d'exemples concrets montre que l'opposition classique entre formel et informel basée sur le niveau des taux d'intérêt des emprunts n'est pas justifiée : à service égal (de la part du prêteur) et à niveau de garantie comparable (pour l'emprunteur) les taux des deux systèmes sont voisins. Après une période interventionniste, le secteur formel a été soumis à un certain nombre de réglementations libérales depuis 1983. En milieu rural cette politique a entraîné une multiplication des banques et des progrès dans la mobilisation de l'épargne. En revanche les crédits distribués ont diminué par leur volume et leur nombre et sont même inférieurs à l'épargne collectée. Le secteur informel remplit ainsi une fonction essentielle au niveau du crédit et de l'assurance en particulier pour les pauvres. Au niveau institutionnel, se pose le problème de la création de services de crédit capables de répondre à la demande rurale, exprimée actuellement par des ménages et des micro-entreprises qui, pour la plupart, ne remplissent pas les conditions nécessaires à l'entrée sur le marché financier formel.

\begin{abstract}
The authors describe the diversity and role of both informal and formal rural financial systems through the analysis of different case studies. They demonstrate that it is not justified to contrast the informal sector with the formal sector on the basis of interest rate levels: for comparable levels of services (by the lender) and guarantee (from the borrower), interest rates charged by both systems are equivalent. Until 1983, the formal financial sector was tightly controlled by interventionist public policies. Since 1983 , the process of deregulation has led to a large increase in the number of banks and to sensible improvements in saving mobilisation. However, the amount and number of loans to the rural sector have decreased and are falling below the level of rural savings. Informal agents are thus playing a decisive role in credit and insurance systems, especially towards the poor. The institutional issue is now to create and develop suitable credit services to match the characteristics of rural demand, which does not meet the minimum requirements to enter the formal financial market.
\end{abstract}


volution
du système financier rural
à Java -Indonésie-

N Tous présentons ici l'ensemble du système financier indonésien qui comprend, comme dans la plupart des pays en développement, les systèmes formel et informel. Le système « formel " correspond aux institutions dont l'existence et le fonctionnement sont reconnus par la législation indonésienne tandis que les acteurs du système « informel » pratiquent une activité de type financier sans contrôle ni intervention directs de l'État.

Notre analyse se base, d'une part sur une série d'interview de responsables d'institutions formelles et, d'autre part, sur une enquête réalisée auprès d'un échantillon aléatoire de ménages entre octobre 1993 et juin 1994, dans les districts de Tasikmalaya et de Bogor à Java-ouest (voir carte en annexe). La région de Tasikmalaya a été choisie pour l'abondance des institutions formelles de crédit et celle de Bogor pour leur faible représentation.

Partant d'un rapide rappel historique sur l'évolution du système financier indonésien, nous présenterons ensuite le paysage financier rural actuel à Java-ouest et l'analyse que nous avons conduite concernant le rôle respectif des secteurs formel et informel. C'est dans ce cadre-là que nous étudierons les conséquences de la libéralisation au niveau de l'offre de services financiers en milieu rural.

\section{Historique du système financier depuis l'indépendance de l'Indonésie}

Nous ne connaissons pas d'études historiques anciennes portant spécifiquement sur les institutions financières et leurs activités. Nous savons seulement que ces institutions étaient déjà très développées dans le passé puisque Lombard (1990) cite des lois sur le commerce de l'argent de la fin du $X^{\mathrm{e}}$ siècle. Dans l'histoire récente et même après l'indépendance en 1945, l'économie reste longtemps contrôlée par les étrangers; le secteur bancaire en particulier est dirigé par des Hollandais et des Britanniques. Les banques seront toutes nationalisées entre 1960 et 1965 . En milieu rural, le système financier échappait au contrôle de l'État et la communauté d'origine chinoise en détenait le quasi-monopole (Dorléans, 1992) (1). L'action du Président Soekarno sur le système financier ne sera pas décisive pour son développement et les dernières années de son gouvernement sont caractérisées par un véritable chaos financier (McLeod, 1992).

1. Les Chinois se spécialisent, encore aujourd'hui, dans le commerce des biens et de l'argent car la loi indonésienne leur interdit la propriété de la terre.
Du point de vue financier, le gouvernement de Suharto va rapidement entreprendre de stabiliser et renforcer le système : la loi bancaire de 1967 définit les principes bancaires à respecter même si chacune des sept banques d'État, les banques de développement et les unités villageoises de prêt sont soumises à leur propre loi. De nouvelles initiatives sont prises en faveur des banques étrangères. En septembre 1970, un décret du ministère des Finances ferme le secteur bancaire à de nouveaux entrants; seules les institutions financières non bancaires pourront officiellement se développer. Ainsi, jusque dans les années 1980 , le système financier formel est essentiellement articulé autour du système national public : les banques d'État distribuent les trois-quarts des crédits octroyés et $90 \%$ proviennent des banques nationales (publiques et privées).

En zone rurale, une part considérable des recettes pétrolières est consacrée, dans le cadre de la Révolution verte, au développement et à la diffusion de nouvelles variétés de semences, à l'amélioration de l'infrastructure des petites exploitations agricoles (en particulier l'irrigation), à la mise en place de services et au financement de subventions pour les intrants. La diffusion des «paquets techniques » est appuyée par le développement d'un large programme de crédits ciblés subventionnés, BIMAS, distribués par les unités villageoises de la Banque Populaire Nationale (BRI Unit Desa) (Booth, 1988). Le réseau sera développé sur l'ensemble de l'Indonésie dans ce but, avec 470 unités en 1970 et 3600 en 1984 (Patten, 1991). Le BIMAS a, en outre, été accompagné ou suivi de différents autres programmes subventionnés (« diversification agricole », « petits investissements », « capital d'entreprise $\gg . .$.$) .$

Il s'agit-là de l'approche " traditionnelle » du crédit (Von Pischke, 1983) où le prêt, subventionné et dirigé, est considéré comme un intrant à la production. Mais, selon une critique répandue (Adams, 1992), les prêts subventionnés ont été plutôt concentrés sur les exploitants offrant des garanties sûres. La politique de la Révolution verte, concentrée sur l'île de Java (Maurer, 1986) a conduit à une polarisation sociale : le revenu et le nombre d'ouvriers agricoles et de petits exploitants a diminué tandis que les revenus relatifs des moyens et gros exploitants ont augmenté (Thorbecke, 1992). Du point de vue du crédit, les défauts de remboursement de plus en plus lourds ont conduit, en 1984, à l'arrêt des programmes subventionnés. Malgré tout, il est incontestable que l'ensemble de ces crédits, transformés dans les faits en transferts forfaitaires, a joué un rôle important dans le succès de la Révolution verte à Java et a permis d'atteindre l'autosuffisance en riz en 1984. 
A partir de 1982-83, la chute des prix du pétrole, la crise internationale ressentie par la diminution des financements extérieurs, certaines faiblesses structurelles (endettement extérieur, chômage, base restreinte de produits d'exportation...) orientent l'Indonésie vers une politique de libéralisation. Le programme d'ajustement, recommandé par le FMI et la Banque mondiale, porte sur les politiques fiscales, monétaires, financières et commerciales.

En particulier, afin de financer son développement, l'Indonésie cherche à mobiliser l'épargne domestique et à développer l'offre de crédit. Dans ce but, la politique de libéralisation dans le domaine financier a été mise en place depuis 1983 pour développer et renforcer un système jusque-là trop dépendant des subventions et des directives de l'État. Ainsi, entre 1983 et 1989, plusieurs mesures de dérégulation (juin 83, décembre 87 , octobre et décembre 88 , mars 89 ) ont conduit à la suppression des plafonds des taux créditeurs et débiteurs, de l'encadrement sectoriel du crédit et de la bonification des crédits de trésorerie; ces mesures visent à encourager le développement du marché des capitaux et à renforcer le secteur financier en intensifiant la concurrence (Thorbecke, 1992).

L'ensemble de ces mesures de libéralisation vise le développement et la diversification du système financier formel indonésien en réduisant les contraintes pour les institutions; les systèmes d'intermédiation sont conduits à mener une transition d'une fonction administrative à une fonction commerciale. La mobilisation de l'épargne, nécessaire à l'échelle macro-économique devient alors une préoccupation pour chaque institution. Au niveau rural, ce sont en particulier les unités villageoises de la BRI qui seront restructurées ou fermées (plus de 1000 unités seront fermées entre 1984 et 1985).

\section{L'offre de services financiers en zone rurale javanaise}

\subsection{Le paysage financier rural : diversité et complexité des formes (voir tableaux 1 et 2)}

Aujourd'hui, l'Indonésie compte sept banques d'État, de nombreuses banques commerciales, banques de développement, banques d'épargne et des centaines de « banques populaires de crédit " (BPR) ainsi que beaucoup d'institutions financières non bancaires (coopératives, centres de mise en gage, projet...) (2). Au niveau des banques nationales, seule la BRI, pour laquelle la reconversion des unités villageoises a été menée avec succès, a traditionnellement un impact important en milieu rural. C'est, de plus, la seule institution rurale qui propose des prêts de long terme et autorise des taux d'endettement élevés. Les autres banques commerciales, publiques ou privées, opèrent peu au niveau des petites unités économiques rurales qui requièrent des prêts de faible volume et à haut risque.

Le système financier informel occupe une place prédominante par la population qu'il concerne en zone rurale. Comme dans la plupart des pays en développement, il est difficile de le cerner complètement et de donner une description fidèle à sa richesse et sa complexité. Ses acteurs (individus ou groupes) ne sont pas toujours clairement identifiables, et ils obéissent à des logiques d'ordre divers : logiques économiques (commerçants), sociales (lien entrepreneur salarié, liens familiaux) ou culturelles (interdit religieux sur la perception d'intérêts monétaires). Les transactions financières sont également com-

2. On définit les projets par le fait qu'ils n'ont pas de statut légal officiel mais qu'ils dépendent d'une autre institution reconnue (banque, centre de recherche, ministère...)

Tableau 1. Caractéristiques des institutions formelles

\begin{tabular}{|c|c|c|c|}
\hline NOM & CADRE JURIDIQUE & ORIGINE DES FONDS & TOTAL CLIENTS \\
\hline $\begin{array}{l}\text { Unités villageoises de la Banque } \\
\text { Populaire Nationale, BRI Unit Desa }\end{array}$ & $\begin{array}{l}\text { Agences de la BRI (banque générale dans le } \\
\text { cadre de la loi bancaire) }\end{array}$ & Fonds propres & $\begin{array}{l}1,8 \text { millions de prêts } \\
\text { en cours }\end{array}$ \\
\hline $\begin{array}{l}\text { Banques locales de développement } \\
\text { de Java Ouest, BKPD }\end{array}$ & $\begin{array}{l}\text { Banque Rurale de Crédit dans } \\
\text { le cadre de la loi bancaire. }\end{array}$ & $\begin{array}{l}\text { Gouvernement provincial, banque de déve- } \\
\text { loppement provinciale }\end{array}$ & 172000 \\
\hline $\begin{array}{l}\text { Services d'épargne et de crédit des } \\
\text { coopératives villageoises, KUD. }\end{array}$ & Coopératives & $\begin{array}{l}\text { Banque coopérative nationale et gouverne- } \\
\text { ment Hollandais. }\end{array}$ & 100000 \\
\hline Unions de crédit & ONG, statut coopératif & $\begin{array}{l}\text { Banque nationale coopérative et prêts entre } \\
\text { Unions }\end{array}$ & 200000 \\
\hline $\begin{array}{l}\text { Projet de lien Banque } \\
\text { formelle-Association villageoise, } \\
\text { PHBK }\end{array}$ & $\begin{array}{l}\text { Projet pilote de la Banque Centrale Indoné- } \\
\text { sienne }(\mathrm{BI})\end{array}$ & Bl et agence de coopération allemande & 20000 \\
\hline $\begin{array}{l}\text { Projet de crédit pour les petits agri- } \\
\text { culteurs; P4K }\end{array}$ & Projet du ministère de l'agriculture & BRI, FIDA, PNUD. & 160000 \\
\hline $\begin{array}{l}\text { Projet de réplication de la Grameen } \\
\text { Bank, KUM }\end{array}$ & $\begin{array}{l}\text { Projet pilote d'un institut indonésien de } \\
\text { recherche en économie rurale. }\end{array}$ & $\begin{array}{l}\text { Centre de développement Asie-Pacifique et } \\
\text { fonds propres }\end{array}$ & 600 \\
\hline Centre de mise en gage & Entreprise publique & Ministère des finances et fonds propres & \\
\hline
\end{tabular}


plexes car fréquemment elles mettent en jeu simultanément des échanges de monnaie, de biens réels et de travail. Nous distinguons pourtant les formes suivantes :

1. Les tontines, arisan en indonésien (association rotative de crédit et d'épargne). Celles des villages étudiés à Java-ouest fonctionnent de façon relativement rudimentaire par rapport à d'autres systèmes décrits en Afrique par exemple. On peut les classer en deux types : les tontines de voisinage (ou tontines « mutuelles » selon Servet (1990) où chaque participant reçoit autant qu'il verse) et les tontines commerciales. Les tontines de voisinage réunissent des groupes de 10 à 40 personnes (souvent des femmes) qui se retrouvent régulièrement pour épargner et tirent au sort le bénéficiaire de la cagnotte. Il n'y a pas mise en vente de cette cagnotte et l'argent de la tontine, qui représente de petites sommes (une centaine de milliers de roupies) est en général consommé. Il existe également de nombreuses tontines spécialisées par exemple dans la vaisselle où la somme apportée à chaque réunion peut correspondre à la valeur d'une tasse; le total de la mise servira à acheter un service complet. La fonction essentielle des tontines est plutôt sociale que financière; elle correspond, en outre, à un modèle de consensus social encouragé par l'État. Il existe aussi des tontines « commerciales " et "financières » (3) gérées par des entrepreneurs ou des commerçants qui s'allient dans un but plus intéressé. Les sommes mises en jeu sont alors plus importantes (de l'ordre de plusieurs millions de roupies) et le tour d'obtention de la cagnotte peut être négocié ou acheté aux enchères afin de satisfaire le membre qui a la meilleure opportunité de placement.

\section{Les associations d'épargne : andil et coopérative scolaire}

Les hommes de certains hameaux se regroupent pour épargner collectivement en vue de la fête du mouton ou de la fête du Ramadan. Ils épargnent de petites sommes (quelques milliers de roupies chaque semaine) pendant plusieurs mois avant l'achat commun d'un ou de plusieurs animaux. La viande est partagée en parts (andil en indonésien) proportionnelles à la mise de chacun, puis, redistribuées aux membres.

Les coopératives scolaires appartiennent au système formel mais leur gestion est entièrement laissée au soin de leurs adhérents. Selon Gentil (1993) elles font partie d'un secteur « intermédiaire ». Leur fonction essentielle est de permettre aux familles d'épargner régulièrement de petites sommes qui serviront à l'achat de matériel scolaire et de l'uniforme des enfants. En théorie, les adhérents ont la possibilité d'emprunter à cette coopérative à des taux relativement bas, voire nuls mais le système de sélection des emprunteurs s'oriente souvent vers les amis ou la famille de l'instituteur responsable de la coopérative. Parmi nos observations, ce système est le seul système financier local qui permet à des individus d'abuser d'un pouvoir monopolistique et conduit à une rémunération négative de l'épargne (les parents épargnent souvent plus que la valeur réelle de l'uniforme que le maître achète à leurs enfants).

3. Dans les tontines commerciales, l'organisateur prélève des frais de gestion mais la cagnotte est tirée au hasard, dans les tontines financières la cagnotte est vendue aux enchères.

\section{Les garde-monnaie}

Ce sont des personnes de confiance qui acceptent de garder de l'argent liquide pour des individus.

\section{Les commerçants}

Les détaillants, épiciers et tout petits restaurateurs (warung) des villages font très souvent crédit (kredit) à leurs clients. Ces crédits à la consommation de première nécessité, de faible volume, sur des périodes courtes, auprès de ménages souvent pauvres, peuvent avoir un coût élevé (jusqu'à $20 \%$ par mois).

On observe également des marchands ambulants spécialisés dans la vente à crédit de vêtements et de vaisselle. La clientèle comme les marchands sont souvent des femmes du village. Les taux pratiqués sont extrêmement élevés (entre 20 et $100 \%$ par mois), mais ces crédits sont également de très faible volume (moins de 100000 roupies) et demandent une gestion très importante car les remboursements sont quotidiens ou hebdomadaires. De façon surprenante, les client(e)s appartiennent à toutes les couches sociales et les biens achetés ne sont pas toujours de première nécessité.

Enfin des commerçants collecteurs des produits agricoles pratiquent couramment l'achat sur pied de la récolte (ijon). Ce système décrit pour le riz (Sturgess et al, 1984) existe également pour certains arbres fruitiers. Un régime de banane est vendu à $3000 \mathrm{Rp}$ deux mois avant sa récolte au lieu de 12000 Rp à maturité. De nombreux agriculteurs préfêrent vendre sur ces « marchés à terme », même s'ils n'y sont pas contraints par un besoin urgent de trésorerie, car ils se mettent ainsi à l'abri des risques de vol, de maladie et de casse; le prêteur assume l'entière responsabilité de la récolte et de la commercialisation. D'autres productions fruitières moins risquées (durians ou papaye) sont rarement vendues à l'avance ou à des taux inférieurs.

\section{Les banques de proximité (bank keliling)}

Leur statut juridique n'est pas toujours clair, coopératives, entreprises privées légales ou non ..., mais elles ont la même dénomination, bank keliling, auprès des ruraux et leur fonctionnement est identique. Un employé démarche les ménages à domicile et leur propose des crédits à remboursement quotidien. Les montants sont faibles en général $(100000 \mathrm{Rp}$ remboursés en 40 jours) et les taux élevés ( $22 \%$ par mois pour cet exemple). On ne demande aucune garantie sauf une photocopie de la carte d'identité (que tout le monde possède à Java), l'utilisation du prêt est libre et le service très rapide.

\section{La mise en garantie de biens (gadai)}

De façon informelle, de nombreux échanges financiers s'opèrent entre individus non professionnels contre mise en garantie de biens productifs (terres, arbres fruitiers, véhicules de transport...). Le propriétaire du bien cède son droit d'usage au créancier pour la durée de l'emprunt. Le créancier exploite ce bien comme il l'entend pendant la durée du prêt et récolte la production qui constitue les intérêts. Comme la durée n'est pas spécifiée et que la production est variable (en particulier pour les arbres fruitiers) les intérêts peuvent varier entre 40 et $100 \%$ par an. Ce système correspond à une forme de placement financier pour les créanciers et une forme de crédit pour les débiteurs. Il est très souple et permet d'emprunter des 
sommes relativement importantes (entre 100000 et 500000 $\mathrm{Rp}$ pour un arbre durian, quelques millions de roupies pour une plantation de papaye). Selon l'enquête de Laure Vilcosqui (1994), portant sur l'ensemble des propriétaires d'arbres durian en gadai d'un village, soit 85 personnes, $51 \%$ des emprunts ont été utilisés pour la consommation immédiate, $12 \%$ ont servi à l'achat de biens de consommation durables (électroménager, rénovation de l'habitat) et $18 \%$ ont été investis. Notons que parmi ces 85 propriétaires, $80 \%$ d'entre eux ont déclaré que cette mise en gadai était pour eux le seul moyen d'emprunter. Cela signifie que les procédures d'emprunt bancaire ont un caractère rédhibitoire pour ces ménages qui dans la majorité des cas pourraient y avoir accès.

Sous le même nom, mais fonctionnant de façon formelle, existent aussi des centres de mise en gage (Pusat Jawatan Pegadaian; voir tableaux) offrant des services de crédit contre le gage de biens non productifs (or, électroménager, véhicules personnels, habits...).

\section{Les prêts personnels sans garanties}

Ces prêts entre membres de la même famille, entre voisins, entre collègues de travail ou entre employeurs et employés sont assez répandus. Ils fonctionnent sans intérêts mais en réalité il existe souvent une contrepartie en travail ou en cadeaux.

Il faut enfin rappeler que les systèmes d'assurance sont sousdéveloppés et qu'à quelques exceptions près, la majorité des familles rurales ne dispose pas d'assurance formelle (aucune assurance sur les enquêtes de Bogor; $10 \%$ sur Tasikmalaya, lorsqu'on compte les assurances-vie, les assurances-maladies...). En cas de besoin urgent elles liquident une partie de leur épargne, s'endettent ou font appel à la famille et à la solidarité villageoise ou du voisinage.

Deux thèses tendent à expliquer la présence du secteur informel (Germidis et al., 1991) : il viendrait pour certains, des carences du secteur formel suite aux politiques financières répressives; pour d'autres, il serait la conséquence du dualisme intrinsèque des structures économiques et sociales de la société rurale. Dans les deux cas, les secteurs formels et informels fonctionneraient sur des segments de marchés différents. Il est également admis que les coûts de ce système pour ses utilisateurs sont généralement usuraires ce qui conduit à l'hypothèse d'imperfection des marchés (crédit cher, gamme de choix limitée en zone rurale...). Notre analyse semble amener à des conclusions moins tranchées.

\subsection{Analyse de la distinction formel/informel}

Malgré leur diversité et apparente incohérence, les services et les taux proposés par les institutions formelles et informelles peuvent être analysés selon les critères d'économie financière. D'après Bottomley (1975) les prêteurs intègrent quatre paramètres à leur calcul avant de fixer leur offre : le risque de non remboursement, les coûts de transactions, le coût d'opportunité du capital et la rente monopolistique. On peut rajouter un cinquième facteur : le degré de parenté ou de voisinage.

Les institutions informelles de type banque de proximité, crédit à domicile, ou prêts des commerçants, pratiquent les taux les plus élevés (4) : les volumes faibles, les remboursements lents et non réguliers, le service de proximité, mais surtout l'absence de garanties impliquent des coûts élevés : coûts d'opportunités des fonds prêtés, en particulier pour les commerçants, gestion des risques basée uniquement sur la pression sociale, coûts de transaction pour les crédits de proximité... Ces prêts sont souvent qualifiés d'usuraires mais l'exemple du projet KUM, dont la vocation sociale semble sans ambiguité, nous prouve que les taux pratiqués par les usuriers ne sont pas aussi scandaleux qu'on veut parfois le dire. Bien que l'organisation du projet bénéficie de l'expérience de la Grameen Bank (Hossain, 1988), les taux pratiqués demeurent élevés (taux effectif de $70 \%$ ). En effet, la distribution de crédits de faible volume et sans garanties implique des coûts de gestion et d'assurance contre le risque. Ainsi, l'essentiel des taux pratiqués pour des prêts sans garanties correspond aux coûts de transaction et à la prime de risque; la part de la rente monopolistique, souvent mise en cause dans les systèmes informels, reste faible.

De même, on peut vérifier la proximité des systèmes formels et informels en analysant les taux pratiqués par les banques régionales, les coopératives villageoises et ceux du système gadai. Ces taux sont comparables et correspondent à des prêts à moyen terme avec garantie matérielle ou nantissement par un fonds d'épargne. Ils sont au moins supérieurs de 10 points à ceux de la BRI car ils incluent des frais financiers supplémentaires dans le cas formel : les banques régionales et les coopératives ne fonctionnent pas sur fonds propres contrairement à la BRI. Dans lc scctcur informel, peut apparaître une légère rente monopolistique liée à la rapidité du service et à la souplesse de l'échéancier. Cependant cette différence ne semble pas excessive (voir graphiquel et tableau 2).

\footnotetext{
4. Le calcul des taux d'intérêt " effectifs " prend en compte (i) les frais d'établissement de dossier, (ii) le coût réel lorsque les intérêts sont calculés sur le principal et non pas sur le montant restant dû, (iii) le manque à gagner d'une épargne obligatoire non ou peu rémunérée. D'autre part, une partie des institutions fonctionne sans mobilisation d'épargne volontaire; les autres rémunèrent l'épargne à des taux sensiblement identiques

(autour de $1 \%$ par mois). II est donc inutile de raisonner en différentiel de taux débiteur et créditeur. Enfin, la durée de ces prêts est majoritairement de 10 ou 12 mois, parfois 6 mois. Seuls les crédits porte-à-porte ou les crédits des commerçants peuvent être plus courts. Ainsi, il est possible, sans introduire trop de biais, de comparer les taux annuels des prêts entre les différentes institutions.
} 


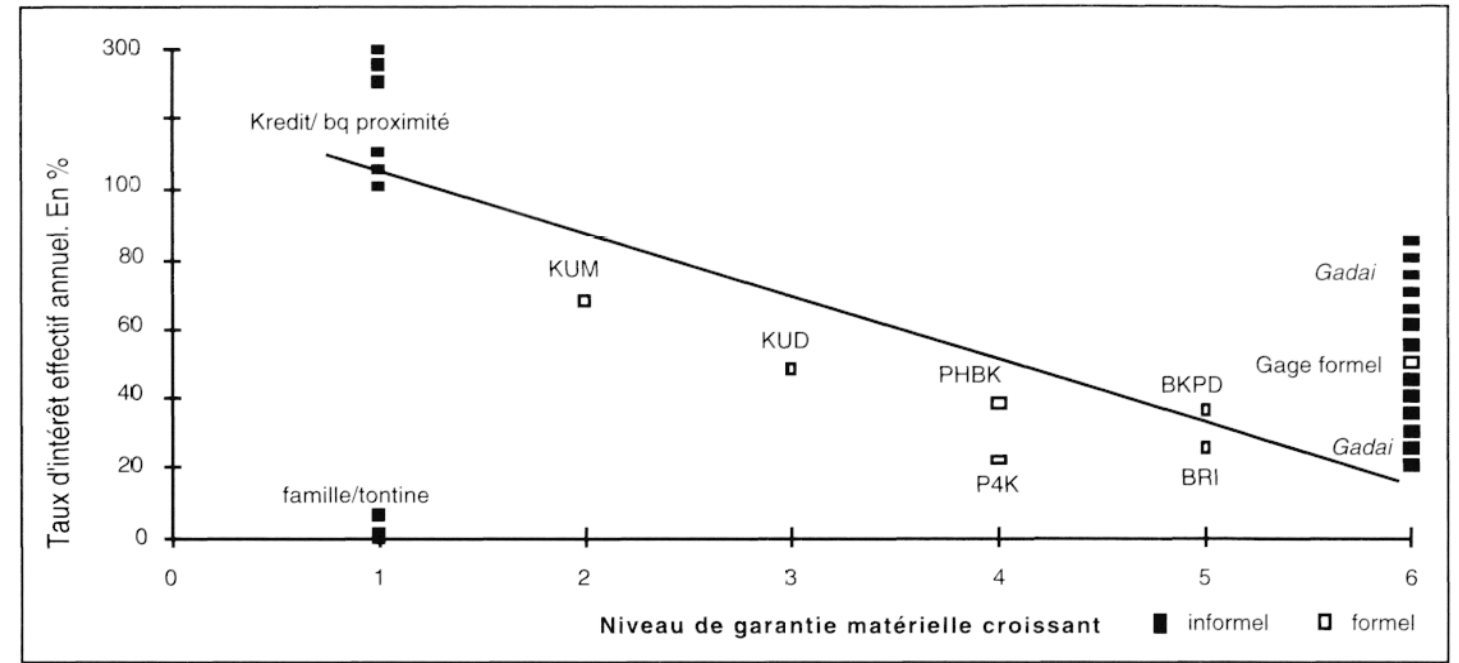

Tableau 2. Caractéristiques des services de crédit -classés par taux décroissant-

\begin{tabular}{|c|c|c|c|c|c|}
\hline Nom & Type de financement & Type de garantie & $\begin{array}{l}\text { Taux } \\
\text { intérêt } \\
\text { annuel } \\
\text { en \% }\end{array}$ & $\begin{array}{l}\text { Montant } \\
\text { Milliers } \\
\text { de } R p\end{array}$ & Durée \\
\hline Banque de proximité & INFORMEL & Pression sociale & 100 à 300 & 5 à 50 & Semaines/mois \\
\hline Kredit & $\begin{array}{l}\text { INFORMEL } \\
\text { Crédit des commerçants } \\
\text { à la consommation }\end{array}$ & Pression sociale & 100 à 300 & 2 à 25 & Semaines \\
\hline KUM & $\begin{array}{l}\text { FORMEL } \\
\text { Crédit individuel par } \\
\text { l'intermédiaire d'un groupe }\end{array}$ & Pression de groupe & 70 & 50 à 300 & 1 an \\
\hline Gadai & $\begin{array}{l}\text { INFORMEL } \\
\text { Crédit avec mise en gage }\end{array}$ & Bien productif & 40 à 100 & 100 à 200 & Année (s) \\
\hline $\begin{array}{l}\text { KUD } \\
\text { Crédit des coopératives } \\
\text { villageoises }\end{array}$ & $\begin{array}{l}\text { FORMEL } \\
\text { Crédit individuel par } \\
\text { l'intermédiaire d'un groupe }\end{array}$ & $\begin{array}{l}\text { Pression de groupe } \\
\text { et garantie collective } \\
\text { (terre) }\end{array}$ & 48 & 50 à 100 & Mois \\
\hline Centre de mise en gage & FORMEL & Bien non productif & 36 à 48 & 2 à 1500 & Mois/1 année \\
\hline $\begin{array}{l}\text { BKPD. Banque locale } \\
\text { de développement }\end{array}$ & $\begin{array}{l}\text { FORMEL } \\
\text { Crédit individuel }\end{array}$ & Preuve de propriété & 36 & 100 à 20000 & Mois/ 1 année \\
\hline $\begin{array}{l}\text { PHBK. Projet de lien } \\
\text { Banque formelle- association } \\
\text { villageoise }\end{array}$ & $\begin{array}{l}\text { FORMEL } \\
\text { Crédit individuel par l'intermédiaire } \\
\text { d'une association villageoise }\end{array}$ & Épargne préalable & 36 & 100 à 1000 & Mois/ 1 année \\
\hline Union de crédit & $\begin{array}{l}\text { FORMEL } \\
\text { Crédit individuel par l'intermédiaire } \\
\text { d'un groupe }\end{array}$ & $\begin{array}{l}\text { Épargne préalable } \\
\text { + pression de groupe }\end{array}$ & 36 & 50 à 1000 & Mois/ 1 année \\
\hline BRI (unités villageoises) & FORMEL. Crédit individuel & Preuve de propriété & 20 à 30 & 25 à 25000 & 1 année \\
\hline $\begin{array}{l}\text { P4K. Projet du ministère } \\
\text { de l'agriculture }\end{array}$ & $\begin{array}{l}\text { FORMEL } \\
\text { Crédit collectif }\end{array}$ & Epargne préalable & 22 & 100 à 250 & 1 année \\
\hline Arisan & $\begin{array}{l}\text { INFORMEL } \\
\text { Association rotative d'épargne } \\
\text { et de crédit }\end{array}$ & $\begin{array}{l}\text { Pression sociale } \\
\text { réseau de solidarité } \\
\text { (voisinage, activité...) }\end{array}$ & 5, nul ou négatif & 10 à 1000 & Mois \\
\hline Famille & INFORMEL & Solidarité familiale & Nul ou négatif & 10 à 5000 & Mois/année \\
\hline
\end{tabular}


Enfin, les cas largement répandus de prêts familiaux ou de voisinage fonctionnent en suivant non plus une logique commerciale mais plutôt en répondant à une fonction sociale d'entraide et de solidarité. Dans ce cas, l'analyse financière précédente n'est pas pertinente pour expliquer des prêts sans garanties, à taux nuls voire négatifs.

Ainsi, la distinction habituelle faite entre systèmes formel et informel basée sur les taux pratiqués par chacun d'eux semble inexacte dans le contexte javanais; il parât plus approprié de s'appuyer sur les coûts de gestion et le niveau de garantie offert par les clients pour comprendre une grande partie des différences observées dans les taux d'intérêt.

Le système financier rural se caractérise par la concurrence des secteurs formel et informel en fonction des garanties requises et donc des risques pour les prêteurs. Ce résultat peut être nuancé par une analyse plus fine des services proposés, en particulier la rapidité d'accès au crédit. On peut alors penser que la libéralisation financière qui implique directement le secteur formel aura des répercussions sur le fonctionnement du système informel.

\section{Conséquences et infléchissement de la libéralisation}

\subsection{Multiplication des banques}

La libéralisation s'est traduite par la multiplication du nombre d'agences et de banques essentiellement privées :

Évolution du nombre de banques et d'agences (entre parenthèses) en Indonésie entre 1983 et 1993

\begin{tabular}{|c|c|c|c|c|}
\hline \multirow[b]{2}{*}{ Banques commerciales } & \multicolumn{2}{|c|}{1983} & \multicolumn{2}{|c|}{1993} \\
\hline & & & & \\
\hline - publiques (1) & 5 & (727) & 5 & (981) \\
\hline - privées & 81 & (337) & 183 & $(2830)$ \\
\hline \multicolumn{5}{|c|}{ Banques de développement } \\
\hline - publiques nationales & 1 & (22) & 1 & (42) \\
\hline - publiques provinciales & 27 & (185) & 27 & (425) \\
\hline - privée & 1 & (1) & 1 & (19) \\
\hline \multicolumn{5}{|l|}{ Caisses d'épargne } \\
\hline - publiques & 1 & (12) & 1 & (43) \\
\hline - privées & 2 & (2) & 2 & (89) \\
\hline \multicolumn{5}{|l|}{ Banques rurales (2) } \\
\hline - banques villageoises (3) & 5642 & & 5343 & \\
\hline $\begin{array}{l}\text { - banques populaires } \\
\text { de crédit (privées) }\end{array}$ & 168 & & 3206 & \\
\hline
\end{tabular}

Données Bank Indonesia, 1994

1. Pour la BRI seules les agences au niveau des districts et sous-districts sont comptabilisées; les unités villageoises ne sont pas prises en compte.

2. Pour les banques rurales, le nombre de banques est équivalent au nombre d'agences.

3. Les banques villageoises sont des unités très petites (Bank Desa Lumbung Desa/ Banques et greniers villageois) mises en place depuis la fin du siècle dernier généralement.

5. Paket january : mot à mot réglementation de janvier

6. Calcul du coût d'opportunité de l'épargne sous forme d'or (hors inflation; $6 \%$ en 1993) :

Solution 1 : achat à 24000 Rp d'1 gramme d'or,
La situation d'un grand nombre de banques demeure fragile. Elles sont sous-capitalisées et le volume de leurs encours est dangereusement élevé (Nasution, 1991). Des faillites exemplaires (banque DUMA en 1992) ont révélé aux épargnants et au gouvernement les limites de cette politique.

Les réglementations du système financier mises en place à partir de 1990 marquent un infléchissement : en janvier 1990 (le pakjan) (5) les crédits directs de la Banque centrale sont supprimés ainsi qu'une grande part des crédits prioritaires subventionnés pour certains secteurs de l'économie. En mars 1991 , les règles prudentielles internationales sont explicitement imposées; la croissance de la masse monétaire est freinée afin de limiter l'inflation.

\subsection{Progrès dans la mobilisation de l'épargne}

La libéralisation du système financier a eu des effets sensiblement différents en zone rurale. La mobilisation de l'épargne rurale, totalement négligée jusque-là, a connu une forte expansion; en particulier, la politique suivie par la BRI à travers le programme SIMPEDES a conduit les unités villageoises à être sur-liquides : à leur niveau, on compte, en décembre 1992, un excédent de 850 millions de dollars US de l'épargne par rapport au crédit (rapport de la BRI, février 1993). Le succès de cette campagne de mobilisation est essentiellement dû au mode de fonctionnement du programme SIMPEDES (plus qu'à ses taux d'intérêt) : retraits illimités, coupons pour une loterie bisannuelle, garantie des dépôts.

Cependant, les enquêtes menées à Tasikmalaya et à Bogor montrent que le potentiel d'épargne rurale n'est pas totalement mobilisé. En effet, à Tasikmalaya la moitié du volume total de l'épargne déclarée (or, dépôts monétaires, arisan) prend la forme d'or, $20 \%$ sont placés dans les coopératives locales et les tontines, et $30 \%$ sont déposés à la BRI. Ces chiffres signifient que $50 \%$ de l'épargne ne pénètrent pas dans le circuit économique, $20 \%$ restent dans le circuit local, tandis que $30 \%$ seulement peuvent intégrer le système financier national.

Si l'on s'intéresse plus précisément aux ménages possédant de l'or en tant que forme d'épargne, on observe qu'ils sont plus riches que la moyenne de l'échantillon. Il est particulièrement intéressant de noter que parmi ce groupe, $64 \%$ des ménages possèdent également une épargne sous forme monétaire. L'or serait un moyen de diversification du portefeuille des ménages les plus riches. Son cours est relativement stable et les gens ont souvent plus confiance en l'or qu'en la monnaie. Aussi préfêrent-ils convertir une partie de leurs économies en or même si le coût d'opportunité par rapport à un placement à la banque est relativement élevé (de l'ordre de $13 \%$ sur un an) (6). L'or est également immédiatement mobilisable car il existe une multitude de commerçants et d'orfèvres. Enfin il se porte en bijoux et permet d'affirmer son statut social.

revente à $22000 \mathrm{Rp}$ un an plus tard.

Solution 2 : placement de 24000 Rp à la BRI à $6 \%$ /an.

Avoir disponible $25440 \mathrm{Rp}$ à la fin de l'année.

Coût d'opportunité $=(25440-22000) / 25440=13,52 \%$ 


\begin{tabular}{l|lccc} 
Le problème de la mobilisation de cette forme d'épargne non \\
intégrée au circuit économique ne se pose donc pas en terme \\
de manque d'information des ménages, ou de difficultés ou
\end{tabular}

Ainsi on voit qu'une partie importante de l'épargne des ménages est redistribuée localement sans intermédiation financière. Le système formel ne semble pas encore aujourd'hui en mesure d'offrir les services de rapidité, de souplesse et de confidentialité qu'offrent les préteurs locaux.

\subsection{Le système formel distribue encore peu de crédit}

En terme de crédit pour Tasikmalaya, plus de la moitié des ménages a, dans les cinq dernières années, contracté un emprunt, formel ou informel et au moment de l'enquête, près de $30 \%$ des familles ont des crédits en cours dont le volume total moyen avoisine les 3 millions de roupies (7). $90 \%$ du volume des crédits en cours au niveau des ménages provient de la BRI, $6 \%$ de la famille et le reste des commerçants et des systèmes coopératifs locaux. Cette écrasante prépondérance de la BRI est un peu nuancée si l'on s'intéresse au nombre de ménages touchés : la BRI finance seulement $35 \%$ des emprunteurs, les systèmes informels (famille, commerçants) en touchent $50 \%$, et les systèmes formels locaux (coopératives) $15 \%$. On observe cependant une forte hétérogénéité entre les sous régions de Java-ouest : dans la région de Bogor, les crédits distribués par la BRI sont extrêmement faibles. La majorité des crédits passe par le système informel via la mise en garantie, le prêt familial, la vente à crédit.

D'une façon générale, le système formel permet l'octroi de volumes relativement importants de crédit (de l'ordre de

7. Notons que si l'on ne compte pas les deux plus gros emprunts (à 10 et 16 millions de roupies à la BAI), la moyenne tombe à moins d'un million quelques millions de roupies) à des entrepreneurs, agriculteurs ou commerçants aisés; les ménages clients de la BRI à Tasikmalaya se distinguent du ménage moyen de l'échantillon par un revenu par membre, un capital foncier et un capital d'entreprise deux fois plus élevés. D'autre part, l'attribution d'un crédit formel est conditionnée par la présentation d'un plan de financement pour une activité productive. En revanche, les créanciers du système informel n'imposent pas d'utilisation spécifique à leurs clients qui peuvent aussi bien emprunter pour la consommation courante, l'achat de biens durables ou l'investissement.

La prépondérance de la BRI sur Tasikmalaya, et la faible contribution des systèmes locaux tendent à montrer que pour l'instant, la politique de libéralisation du système financier est encore suivie de peu d'effets tangibles malgré l'apparition d'institutions financières dans le district (projets PHBK de la Banque centrale, projet $\mathrm{P} 4 \mathrm{~K}$ du ministère de l'Agriculture, Unions de Crédit...). Des conclusions semblables ont été tirées au cours d'études précédentes menées par la Banque centrale dans deux districts (Cianjur, Java-ouest et Boyolali, Java central) : poids de la BRI, banques populaires absentes (Cianjur) ou peu représentées.

\section{Infléchissement de la réglementation}

Au niveau macro-économique, il faut rappeler que les zones rurales ne bénéficient plus des larges volumes de crédit subventionnés distribués par différents programmes nationaux du gouvernement. Par ailleurs, les données de la Banque centrale (rapport mensuel de mars 1992) montrent que pour 1990-91, les banques nationales distribuent toujours $90 \%$ des crédits et que la part des banques locales n'a pas évolué. La part des banques d'État a été réduite de 75 à $53 \%$ entre 1981 et 1991, or les banques commerciales privées se tournent peu vers les zones rurales. Cette évolution s'est donc traduite par une chute relative du volume de crédit destiné aux zones rurales.

Depuis 1990, une plus grande attention est portée aux zones rurales puisque, suite aux réglementations du Pakjan, chaque banque nationale doit obligatoirement distribuer $20 \%$ de son portefeuille sous forme de crédits aux petites entreprises. D'autre part, $5 \%$ du profit des entreprises publiques doivent être alloués à la recherche ou à ces petites entreprises. La plupart de ces fonds sera distribuée à travers la BRI par un programme spécifique de crédit. Étant donné l'excédent d'épargne observé dans les unités villageoises de la BRI, on peut s'interroger sur la perspicacité de cette mesure. En effet, le facteur limitant la création d'entreprises en milieu rural ne semble pas l'absence de capitaux, mais plutôt, d'après Collier (1993), un manque de formation adéquate.

En bilan, la nouvelle loi bancaire (loi $n^{\circ} 7$ de 1992) synthétise l'ensemble des régulations dictées depuis juin 1983 combinant l'orientation vers une libéralisation du système financier avec les restrictions et règles prudentielles nécessaires pour un développement sain.

Afin de mieux contrôler l'évolution des institutions financières, cette loi impose à chacune de se conformer d'ici 1996 à l'un des deux types de banques suivant : « banque générale » et « banque populaire de crédit ». Ces réglementa- 
tions vont simplifier le paysage financier mais risquent de freiner le développement des petites institutions rurales (les banques de développement local, les projets de crédit aux pauvres et les systèmes d'assurance) tenues à se soumettre à des règles bancaires strictes.

\section{Conclusion}

Le système financier indonésien se caractérise donc par un foisonnement d'institutions, formelles et informelles, étroitement imbriquées et souvent en concurrence. Elles proposent une large gamme de services d'épargne et de crédit en zone rurale. Paradoxalement, le secteur des assurances, au niveau formel, reste quasiment inexistant.

Le système informel de crédit fonctionne essentiellement comme un système de crédit à la consommation et pallie ainsi les lacunes du système formel d'assurances : il sert en général à couvrir les risques sur le revenu (chômage, intempéries...) et les besoins urgents de la famille (maladie, accident, décès...). Le système formel au contraire impose généralement une utilisation du prêt à des fins productives. Ainsi, la distinction formel/informel n'est plus justifiée par des différences de taux d'intérêt mais par les caractéristiques du service offert (proximité, grande souplesse, rapidité, liberté d'utilisation du prêt). Les taux d'intérêt, indépendamment de la reconnaissance juridique, sont davantage liés à des prises de risque et donc au niveau de garantie associé à chaque prêt.

Le système informel conserve un rôle indispensable; si les plus riches peuvent se tourner indifféremment vers les systèmes formels ou informels, les ménages sans garantie ont plutôt recours par nécessité à l'informel. Au-delà des critiques souvent formulées à son encontre, ce secteur acquiert une légitimité (assurance, consommation, services aux plus pauvres...) en particulier nécessaire dans le cadre de la libéralisation du secteur financier formel.

La politique de libéralisation a entraîné, d'une part une meilleure mobilisation de l'épargne et, d'autre part, une redistribution des crédits vers les zones urbaines aux dépens des zones rurales. La polarisation sociale et le déséquilibre apparent de la distribution de capitaux ont incité l'État à mettre en place des régulations adaptées spécifiquement au monde rural, encourageant le retour des fonds vers les campagnes. La pertinence de ces mesures est discutable car le monde rural dispose déjà d'une épargne formelle et informelle importante. La question n'est pas d'apporter de nouveaux fonds mais plutôt de susciter l'investissement. Pour cela, il faudrait adapter l'offre de services formels à la demande en imaginant par exemple de nouveaux systèmes de caution non liés à la propriété foncière, en diminuant les coûts de transaction pour les clients ou, enfin, en ne conditionnant pas l'obtention d'un prêt à une utilisation particulière.

\section{Sandry DURY, ENSAM-INRA}

\section{Cécile LAPENU, ENSAM-CIRAD.}

Les auteurs remercient MM. Benoît-Cattin, $R$. Lifran, $P$. Rio et l'équipe Fermat de I'INRA-ESR pour leur soutien dans l'élaboration de ce travail, ainsi que $M$. Tauzin et F. Richard pour leur appui technique et Ch Cibenel.

\section{BIBLIOGRAPHIE}

Adams WD. Comment établir des marchés financiers ruraux durables. Actes du Séminaire International Finance et Développement Rural, CIRAD, 1992, pp. 11-16.

Adams WD, Fitchett AD. Informal finance in low-income countries. Westview press,1992, 393 p.

Booth A. Agricultural development in Indonesia. SouthEast Asia Publication Series, 1988, $n^{\circ} 16,290$ p.

Bottomley A. Interest rate determination in underdeveloped rural areas. American Journal of Agricultural Economics, 1975, pp 279-291.

Collier WJ, Santoso K, Soentoro, Wibowo R. A new approach to rural development in Java : twenty five years of village studies. Seminar proceedings, Institut Pertanian Bogor (Indonésie), 1993.

Dorléans B. L'Indonésie, les incertitudes du décollage économique.Etudes de la Documentation Française, Paris, 1992, $197 \mathrm{p}$.

Gentil D, Fournier Y. Les paysans peuvent-ils devenir banquiers ? Epargne et crédit en Afrique. Syros, Paris, 1993, $271 \mathrm{p}$.

Germidis D, Kessler D, Meghir R. Systèmes financiers et développement : quel rôle pour les secteurs financiers formel et informel ?. Paris, OCDE, 1991, 276 p.

Hossain M. Credit for alleviation of rural poverty : the Grameen Bank in Bengladesh. International Food Policy Research Institute, Bangladesh Institute of Development Studies, 1988, $n^{\circ} 66,89 \mathrm{p}$.

Lapenu C. Innovations institutionnelles en faveur des populations rurales dans le système financier indonésien. CIRAD-SAR, 1992, n 49/93, 85 p.

Lombard D. Le carrefour javanais. Essai d'histoire globale. Edition de l'Ecole des Hautes Etudes en Sciences Sociales, Paris, 1990.

Tome 1. Les limites de l'occidentalisation. 267p.

Tome 2. Les réseaux asiatiques. $423 p$.

Tome 3. L'héritage des royaumes concentriques,. 337p.

Mac Leod RH. Indonesia's new banking law. Bulletin of Indonesian Economic Studies, 1992, 28 (3), pp 107-129.

Maurer JL. Modernisation agricole, développement économique et changement social. Le riz, la terre et l'homme à Java. PuF, 1986, 322p.

Nasution A. Survey of recent developments. Bulletin of Indonesian Economic Studies, 1991, 27 (2), pp 3-43.

Patten RH. Rosengard JK. Progress with profits. The development of rural banking in Indonesia. San Francisco (USA), ICs Press, 1991, $114 \mathrm{p}$.

Servet JM. Pratiques informelles d'épargne dans les pays en développement. Cahiers « Monnaie et financement », 1990, $\mathrm{n}^{\circ}$ 19, pp 204-233.

Sturgess NH, Wijaya H, Dow N. Usufruct and usury : an analysis of land leasing in East Java. Australian Journal of Agricultural Economics, 1984, 28 (1), pp 15-32.

Thorbecke E. Ajustement et équité en Indonésie. OCDE, Paris, 1992, 274 p.

Vilcosqui L. L'arbre, support de garantie dans le système de crédit informel indonésien : le gadai. Mémoire CNEARC, 1994, $80 \mathrm{p}$. 
VON PISCHKE JD, ADAMS WD, GORDON D. Rural financial markets in developing countries. Their use and abuse. Economic Development Institute of the World Bank, 1983. 430p.

World bank. World development report 1989. Oxford University Press. 1989.

\section{ANNEXES}

\section{L'Indonésie}

A part quelques voyageurs privilégiés et certains défenseurs des droits de l'homme, peu de français connaissent l'Indonésie. Ce pays est pourtant le plus grand archipel du monde et se place au cinquième rang mondial pour sa population (190 millions d'habitants). Le peuplement se répartit de façon inégale entre les îles : la densité démographique de Java (120 millions d'habitants) cst de 800 habitants par $\mathrm{km}^{2}$ tandis que celle de Bornéo atteint 10 habitants par $\mathrm{km}^{2}$.

Politiquement, la stabilité régne puisque depuis l'indépendance en 1945, seuls deux présidents, Sukarno (1945-1965) et Suharto (1966-?) ont dirigé le pays. L'histoire récente montre une croissance importante du PIB (en moyenne 5,8 \% sur les années 1984-1992, en valeur constante 1983), du niveau de vie moyen, du taux de scolarisation et un contrôle des naissances de plus en plus efficace. Grâce au contrôle du taux d'inflation à travers l'adoption de politiques fiscale et monétaire prudentes, la monnaie a pu rester relativement stable (en 92-93, dépréciation de 2,1\% par rapport à un panier des monnaies des principaux partenaires commerciaux) et totalement convertible. Depuis 1984, l'inflation n'a jamais dérapée au-delà d'un pourcentage à un seul chiffre.

Ce développement s'est réalisé en partie grâce à des recettes pétrolières réinvesties dans différents secteurs à l'intérieur du pays. L'agriculture, en particulier, a bénéficié de ces ressources. En 1984, grâce à une forte volonté politique exprimée à travers une série de programmes et de plans, l'Indonésie, qui était connue comme l'un des plus grand pays importateurs de riz, est parvenue à l'autosuffisance. Dans les années 1970-80, les facteurs-clés de cette réussite reposent sur les progranmes de vulgarisation auprès du plus grand nombre (BIMAS) et d'intensification (INSUS) qui impliquent la diffusion de variétés améliorées, l'investissement pour l'irrigation, une politique de prix favorable et des subventions pour des intrants. La production de riz a augmenté de plus de $70 \%$ durant les dernières décennies, passant de 26 millions de tonnes en 1973 à 45 millions de tonnes en 1991. La politique s'est infléchie durant les années 1980 avec une diminution relative des subventions aux intrants et une volonté de diversification des productions. En 1991, l'agriculture et la forêt représentaient $20 \%$ du produit intérieur brut et $24,5 \%$ des exportations non pétrolières. Aujourd'hui à Java, il semble que la riziculture atteigne ses limites en terme de production : ni les rendements en riz avec les variétés actuelles ( 7 tonnes par ha en 1992) ni les surfaces cultivées ne peuvent augmenter (Collier et al., 1993). Le maintien de la croissance en zone rurale qui compte environ $50 \%$ de la population passe actuellement par une diversification des activités dans le secteur agricole et non agricole. En dehors du fort développement "spontané » des activités de transformation et de commerce, le gouvernement encourage actuellement toute création d'entreprise en milieu rural. Selon nos observations et celles de l'équipe de Collier (1993), $50 \%$ des familles rurales ont un de leurs membres au moins qui travaille hors du secteur agricole. A titre indicatif, sur Java-ouest, les dépenses moyennes par tête et par mois en zone rurale s'élèvent à $20000 \mathrm{Rp}$ pour l'alimentation, et $10000 \mathrm{Rp}$ pour les dépenses non alimentaires.

\section{L'indonésie. Situation des terrains d'enquête}

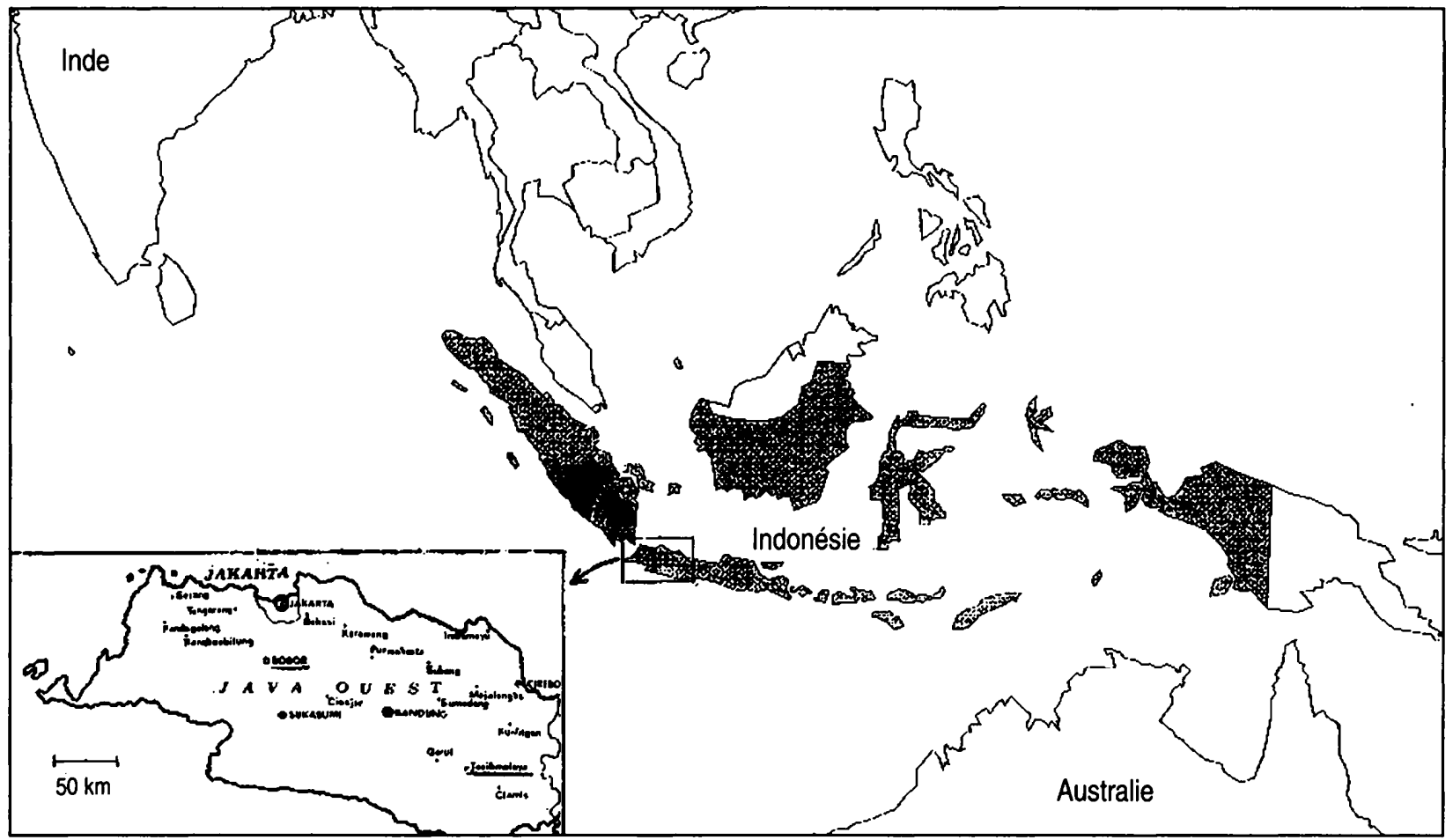




\section{Exemple de budget de ménage}

Tarmidi, jeune agriculteur, père de trois enfants, est l'un des gros propriétaires fonciers de Nagrak, village situé à 15 $\mathrm{km}$ de Bogor, car il possède environ 1,5 hectare de terre arable ce qui représente quelques 230 millions de roupies. La superficie moyenne des exploitations agricoles de Java est de 0,5 ha et les ménages propriétaires de terres agricoles représentent moins de $25 \%$ de l'ensemble des ménages dans le village étudié. Il cultive « lui-même » (avec des journaliers) plusieurs parcelles en papaye et en manioc. Ces productions sont facilement commercialisées vers les villes avoisinantes (Bogor et Jakarta). Il a donné en métayage une parcelle que le métayer cultive en riz. L'épouse de Tarmidi est commerçante de vêtements à crédit (capital total de $300000 \mathrm{Rp}$ ). Les deux époux ont un revenu annuel d'environ 3,7 millions de Rp, ce qui place leur ménage au dessus de la moyenne (2,3 millions par an, soit environ $40000 \mathrm{Rp}$ par personne et par mois).

Comme beaucoup de chefs de familles du village, chacun à la hauteur de ses moyens, Tarmidi est impliqué dans un nombre impressionnant de transactions financières et a diversifié ses actifs autant que possible. Au mois de novembre 1993, son portefeuille se composait de créances avec garantie et sans garantie matérielle, de parts dans différentes associations, de dettes et de plusieurs actifs réels.

Contre trois arbres durians pris en gadai il a prêté $600000 \mathrm{Rp}$ à un voisin pour les funérailles d'un parent. Le montant du prêt a été négocié car ce voisin réclamait $800000 \mathrm{Rp}$. La durée du prêt dépend de l'emprunteur mais le contrat, oral, précise qu'il faut une production minimum de $450000 \mathrm{Rp}$ avant le remboursement. Suivant le moment et le montant réel de la production (ces arbres fruitiers ont une production aléatoire en volume et dans le temps) le taux d'intérêt implicite peut varier grandement autour des $75 \%$ théoriques (production la première année après l'emprunt égale à $450000 \mathrm{Rp}$ et remboursement après un an). $\mathrm{Si}$ la production atteint $800000 \mathrm{Rp}$ la première année et si le remboursement est immédiat alors le taux serait de $110 \%$. En revanche si la production est nulle pendant trois ans puis égale à 450000 , le taux annuel atteindra seulement $20 \%$. Ainsi le taux d'intérêt (non monétaire) de ce type de gadai (sur durian) bien qu'élevé en moyenne est très risqué. Tarmidi a également des créances sans garantie matérielle de l'ordre de 2 millions au total auprès de 10 personnes différentes. Suivant la durée du prêt (qui dépend de l'emprunteur mais qui excède rarement un an), le taux d'intérêt varie entre 30 et $50 \%$. L'intérêt monétaire a dans ce cas une variance plus faible.

Tarmidi participe à une tontine (arisan) de 20 personnes qui déposent chaque mois 50000 roupies, il est membre d'une association d'épargne religieuse (andil) qui collecte $1000 \mathrm{Rp}$ par semaine pendant 10 mois pour l'achat d'un boeuf à la fête du Ramadan. Enfin, le ménage possède de l'épargne thésaurisée sous une forme d'or (750000 Rp) d'animaux (200000 Rp), de monnaie (300000 Rp).

Bien que ce ménage soit l'un des plus riches du village, on notera que ses liquidités représentent une faible partie de son patrimoine et qu'il est régulièrement obligé d'emprunter (en général à l'acheteur collecteur) car ses revenus sont irréguliers. Il a récemment acheté une télévision 500000 Rp à crédit, payable en 10 fois et Tarmidi doit actuellement $25000 \mathrm{Rp}$ à ses beaux-parents car il était à court de liquidités le mois dernier.

Cet exemple particulier représente assez bien à notre avis la situation de la majorité des ménages ruraux de Java : la plupart d'entre eux ont un taux d'endettement extrêmement faible (en moyenne $95 \%$ de leur capital est financé par des ressources propres) et quels que soient leur niveau de richesse et leur activité, ils possèdent en général peu de liquidités et diversifient leurs actifs. Nous n'insistons pas ici sur les supports non financiers de l'épargne et du patrimoine mais il est clair que de nombreux ménages utilisent par exemple l'or et les animaux plutôt que des actifs financiers pour gérer leur budget au cours du temps. Cela implique des coût de transactions importants qu'il faudrait comparer aux avantages (en terme d'utilité, de revenu, de diminution du risque...) procurés par ces actifs.

\section{Utilisation des prêts}

(D'après les données d'enquête sur le projet KUM, réplication de la Grameen Bank; 30 emprunteurs; Lapenu 1992) Rappel : il s'agit de prêts de faible montant (30 à $75000 \mathrm{Rp}$, généralement $50000 \mathrm{Rp}$, plus importants pour les $2^{\mathrm{e}}$ et $3^{\mathrm{e}}$ prêts) à remboursement hebdomadaire sur un an.

On observe la répartition suivante :

\begin{tabular}{|c|c|c|c|c|c|c|}
\hline & Femm & & & Homm & & \\
\hline & Product & Educat & Consom. & Product & Educat & Consom. \\
\hline 424 & & 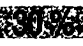 & & & & \\
\hline${ }^{\mathrm{e}}$ prêt & $60 \%$ & $10 \%$ & $30 \%$ & $70 \%$ & $5 \%$ & $25 \%$ \\
\hline
\end{tabular}

L'utilisation du prêt à des fins productives permet généralement de pratiquer plus régulièrement ou avec un plus grand volume une activité qui existait déjà. Il s'agit souvent d'une activités commerciales (vente de produits alimentaires transformés sur place - pâtisseries, préparations salées, jus de fruits...- et vendus dans un petit local, warung ou avec du matériel ambulant, pikulan; achats de tissus, vêtements... à Bogor ou Jakarta qui sont revendus, souvent à crédit au sein du village; vente de produits agricoles de l'exploitation), d'activités agricoles (achat de semences, engrais, petit bétail...) ou de services (taxi moto...). Les dépenses pour les frais de scolarité des enfants correspondent à une utilisation fréquente, davantage marquée pour les prêts aux femmes.

Lors des $2^{\mathrm{e}}$ et $3^{\mathrm{c}}$ prêts, le pourcentage destiné à l'activité productive décroît mais la part absolue reste cependant sensiblement constante (montant nécessaire à maintenir l'activité); en contrepartie, la part à la consommation augmente, utilisée pour améliorer le confort de l'habitat (matériel de construction, électricité, radio, TV...) ou pour l'achat d'or sous forme de bijoux (forme d'épargne).

Ainsi, malgré la théorie du projet où le crédit doit être exclusivement utilisé pour une activité productive, l'observation montre que la pression est forte pour des activités dont la productivité n'est pas directe. 